\title{
Relating Cognitive Models of Design Creativity to the Similarity of Sketches Generated by an Al Partner
}

\author{
Pegah Karimi ${ }^{1}$, Nicholas Davis ${ }^{1}$, Mary Lou Maher ${ }^{1}$, Kazjon Grace ${ }^{2}$, Lina Lee ${ }^{1}$ \\ ${ }^{1}$ University of North Carolina at Charlotte, ${ }^{2}$ The University of Sydney \\ \{pkarimi, ndavis64, m.maher, llee52\}@uncc.edu,kazjon.grace@sydney.edu.au
}

\begin{abstract}
This paper presents and evaluates a new method for inspiring creativity in a co-creative design system. The method uses a computational model of a conceptual shift based on clustering of deep features from a database of sketches. The co-creative sketching tool maps a user's sketch to a sketch of a distinct category that has high, medium, or low visual and semantic similarity. We hypothesize that the degree of similarity between the user's and the system's sketches is associated with a range of cognitive models of creativity in a design context. We report on the findings of an empirical study that analyzes different design scenarios in which the user sketches in response to a proposed conceptual shift. The findings show that how similar the computational agent's sketch is to the user's original sketch is related to the presence of three types of design creativity in the user's response: combinatorial, exploratory, and transformational.
\end{abstract}

\section{Author Keywords}

Design Creativity, Sketching, Collaboration, Co-Creativity

\section{CCS Concepts \\ -Human-centered computing $\rightarrow$ Collaborative interac- tion;}

\section{INTRODUCTION}

Human-machine collaboration has the capacity to augment creativity in a number of ways. During collaboration, ideas emerge from both users and the system that can support and inspire creativity [25]. As collaborators make unexpected and novel contributions, their product can lead to new artifacts that otherwise they might not have achieved individually [25]. A new branch of intelligent interactive systems has emerged, called co-creative systems [8], that features human users creatively collaborating with AI agents. These systems have been applied in various domains, including art [9, 15], music [2], games [22, 20], and robotics [14]. In all cases, these systems are designed to help encourage creative thoughts for both novices and experts. These new types of co-creative systems are proposed as ways to increase the level of user's engagement with the system, support innovation and inspiration,

Permission to make digital or hard copies of all or part of this work for personal or classroom use is granted without fee provided that copies are not made or distributed for profit or commercial advantage and that copies bear this notice and the full citation on the first page. Copyrights for components of this work owned by others than ACM must be honored. Abstracting with credit is permitted. To copy otherwise, or republish, to post on servers or to redistribute to lists, requires prior specific permission and/or a fee. Request permissions from permissions@acm.org.

C\&C '19, June 23-26, 2019, San Diego, CA, USA

(C) 2019 ACM. ISBN 978-1-4503-5917-7/19/06 . .\$15.00

DOI: https: //doi .org/10.1145/3325480.3325488 motivate the user to continue the task, and help users achieve shared goals.

In this paper, we describe the computational processes in a co-creative sketching tool meant to help designers generate and iterate on new design ideas. We have developed a computational model for making conceptual shifts [18], which can analyze and extract visual and semantic features from the user's sketched object and then identify a relevant object to display in order to encourage user creativity. The model takes the user's sketch and compares it with a sketch of another category with a measure of semantic and visual similarity. Semantic similarity is a measure of the distance between the meaning of the two categories using a word embedded model. Visual similarity is a measure of the distance between the visual features of the two sketches. Leveraging the amount of semantic and visual similarity can encourage designers to explore new design ideas and lead to more creative outcomes.

Sketching in design serves as a way to communicate ideas and explore possible design solutions. Designers often use physical sketches to represent their ideas in the early stage of their design task. These sketches can later be modified, built upon, or explored in another design task. Computational support for sketching has been explored in some co-creative systems, such as the Drawing Apprentice [10], which recognizes the user's sketch and maps it to a sketch of the same conceptual category. In our work, the conceptual shift model maps the user's sketch into a sketch of another conceptual category with which the current sketch shares structural characteristics and belongs to the same contextual space. We posit that moving from one concept to another can serve two purposes: 1) inspire the user's creativity in a co-creative design context and 2) study different types of design creativity associated with the user's response.

We report on a Wizard of Oz user study to explore how our proposed model affects the design creativity of users. We investigate if our co-creative sketching tool can inspire the user's creativity by helping them develop novel and creative ideas for their initial design. We intend for our tool to support different forms of creativity, such as combinatorial, exploratory, and transformational, depending on the parameters selected for the operation of the model. Combinatorial creativity involves adding two sets or subsets of design ideas together; exploratory creativity is when we explore alternative design ideas in the same design space and transformational creativity involves changing the overall search space in which the design creativity occurs [3]. We explore how the degree of similarity of the sketch presented to the designer, both semantically 
and visually, is correlated with the three forms of creativity presented above. Our initial hypothesis is that there is a correlation between high similarity and combinatorial creativity, intermediate similarity and exploratory creativity, and low similarity and transformational creativity.

\section{RELATED WORK}

Computational co-creative systems are a blend of computational creativity and creativity support tools. Computational creativity arose from within AI with the goal of developing computational models that generate creative artifacts without human intervention $[6,31,24,30]$. Conversely, creativity support tools are technologies that encourage the user's creative thoughts and help them to be productive [11, 26, 27, 29]. By combining concepts from computational creativity and creativity support tools, we can develop computer programs that collaborate with human users on a shared creative task. Co-creative systems can adopt different roles to support human creativity, such as nanny, coach, pen-pal, and colleague [21]. The co-creative sketching tool presented here can be considered a computational colleague that designs alongside users by utilizing a computational model of conceptual shifts [17] to inspire creativity.

Conceptual shifts in design occur when a sketch of one category is perceived as belonging to a different category [18]. Identifying conceptual shifts is an important step of the creative process as they entail re-interpreting an idea or a concept and, in doing so, identifying a potential new avenue for exploration. Presenting conceptual shifts has the potential to foster creativity by allowing users to perceive sketches in different ways. Suwa and Tversky [28] describe two different modes of perception used in design: seeing-that and seeing-as. Seeingthat is related to the functional type of perception, in which the concrete properties of a sketch and the role they play in the overall design are considered. By contrast, seeing-as is an interpretative process where elements of a sketch can be viewed through multiple perspectives, such as seeing a collection of shapes in an architectural drawing as a bridge. Engaging with conceptual shifts could help designers to become accustomed to the seeing-as mode of perception by showing how drawn structures could relate to a variety of highly distinct objects.

Additionally, identifying conceptual shifts can enhance the process of analogy making, which is known to be critical in design reasoning [12]. Models of visual analogy serve as a kind of intermediary, in which two semantically distinct objects could be recognized similarly [7]. During a design task, analogies are made between the elements of a design space. In the visual domain, designers often make analogies by associating a source sketch with a target sketch. This form of association can help designers produce novel variations of their initial design and can lead to the discovery of previously unexplored aspects of the creative space.

One important factor for studying design creativity is the ability to distinguish between different types of creativity. Boden describes three forms of creativity: combinatorial, exploratory, and transformational [4]. Combinatorial creativity happens when two sets of design concepts or a subset of them are combined together. Exploratory refers to developing new ideas through traversing an established conceptual space. Transformation involves changing or altering the current design space such that new possibilities exist. The types of conceptual shifts introduced by a co-creative sketching tool have the capacity to result in these three types of creativity, in which designers can establish fluid boundaries between sketch categories.

\section{CONCEPTUAL SHIFT MODEL FOR A CO-CREATIVE SKETCHING TOOL}

Our model for identifying conceptual shift categories consists of two components: visual similarity and semantic similarity. Visual similarity is determined by selecting sketches that share the same underlying structural characteristics. The visual similarity component is balanced by a semantic similarity component which ensures that the two sketch categories are semantically related to each other. We have implemented this model in the domain of human drawn sketches. In this section, we describe our dataset and the methods for computing semantic and visual similarity between sketches.

\section{Dataset}

In our sketching tool, the system selects an example from a large-scale dataset called Quick, Draw! (QD) [16], which contains 345 categories with an average of 120,000 human drawn sketches per category. The dataset was created during an online game, where players were asked to draw a particular object within 20 seconds. The sketch data for this dataset is available both as a low-resolution pixel image and as a vector image. We have developed our conceptual shift model by utilizing the vector image as it encodes the movement of the pen and is closer to how humans draw compared to the pixel image. The vector image represents the coordinates of the strokes associated to each sketch, as well as a binary number that indicates if the pen is lifted from the paper or not.

\section{Visual Similarity}

To compute visual similarity, we encode each sketch as a sequence of pen strokes. Given a user's sketch, we identify the candidates for conceptual shift through two different processes: learning and clustering. The learning step represents each sketch by extracting their features using a deep neural network model, Convolutional Neural Network - Long ShortTerm Memory (CNN-LSTM) [5]. The model has three onedimensional convolutional layers and three LSTM layers. We train the model on QD dataset using 30,000 training samples and 10,000 validation samples per category for 1.5 million training steps. Observation shows that after 1 million training steps, the accuracy, measured as true positive rate, reaches almost 74\% and remains approximately the same afterwards.

The next step, which is clustering, separates the sketches of a category into different clusters, each with its own label. This step ensures that sketches of a category that have the same visual information are grouped into one cluster. Clustering is done by using a k-means algorithm and employing the elbow method to determine the optimal number of clusters. The user's sketch is then assigned to one of the clusters of its category by selecting the minimum L 2 distance between its feature-vector and the centroid of the clusters associated with 


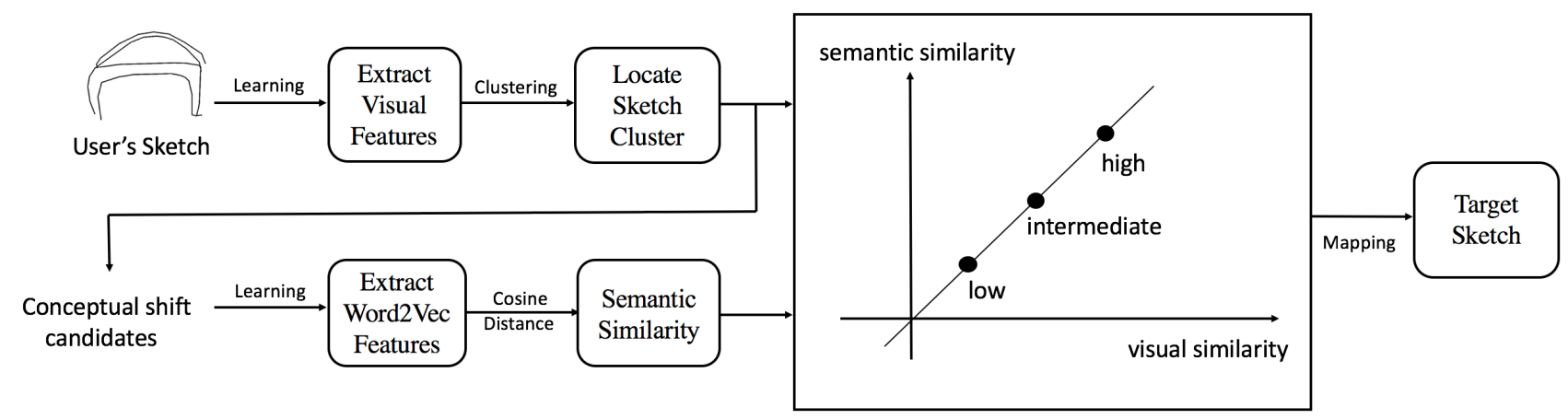

Figure 1. An overview of the algorithm for selecting the target sketch based on the visual and semantic similarity components.

the sketch category. Based on the identified cluster, we determine the top most similar sketches from distinct categories as candidates for conceptual shifts. For more implementation details refer to [19].

\section{Semantic Similarity}

We encode the category names from the set of conceptual shift candidates using the word2vec word embedding trained on the Google news corpus [23]. The word2vec embedding is a feature-vector with a dimension of 300 , which depends on the context in which the word appears. The similarity is defined as the cosine distance between the two feature vectors. We compare the distances between the category names of both the user's input and the conceptual shift candidates to understand the degree of similarity between sketched categories. Smaller distances indicate that the two sketch categories are more likely to appear in the same context, while larger distances show that the two categories are less associated with each other.

\section{Quantify Similarity}

To quantify visual and semantic similarity between two sketches, we first normalize the distances in the feature space described above. For the visual component, this will be $1-d_{v}$, where $d_{v}$ is the L2 distance between centroids normalized across all the conceptual shift candidates. For the semantic component, it will be $1-d_{c}$, where $d_{c}$ is the cosine distance between the vector representations of the category names. In order to measure similarity as a single component, we then select potential conceptual shift categories that have visual and semantic similarity values close to each other. Among the top 20 most visually similar categories, we select the ones for which the difference between visual and semantic similarity is below 0.05 as potential candidates. After selecting potential candidates, similarity is computed as the average of semantic and visual similarity. This corresponds to a single value for the similarity component between the user's sketch and the selected candidates.

Figure 2 shows the semantic and visual similarity values between the "bridge" category and the corresponding top 20 potential conceptual shifts. As it is shown, in some instances the semantic and visual similarity values are very close to each other, indicating that there are some clusters of the two sketch categories that share the same amount of visual and semantic information. In some other instances, however, the semantic and visual similarities are very different, indicating that the two sketch categories have some instances that share the same visual information but are less likely to appear in the same context or vice versa. Example of this is a potential cluster of "bridge" and a potential cluster of "ladder." As Figure 2 shows, the semantic similarity value for the two categories is high, whereas the visual similarity is low, showing that there are no instances of the two categories that share high level of visual information despite belonging to the same contextual space.

The user's sketch is then mapped to the target sketch through the selection of conceptual shift candidates based on three different levels of similarity: high, intermediate, and low. As the changes in the visual similarity values are varying smoothly, we conventionally classify high similarity as the values falling in the top 33rd percentile of the similarity distribution, intermediate similarity as the values between the 33rd and the 66th percentile, and low similarity for the remaining values. Figure

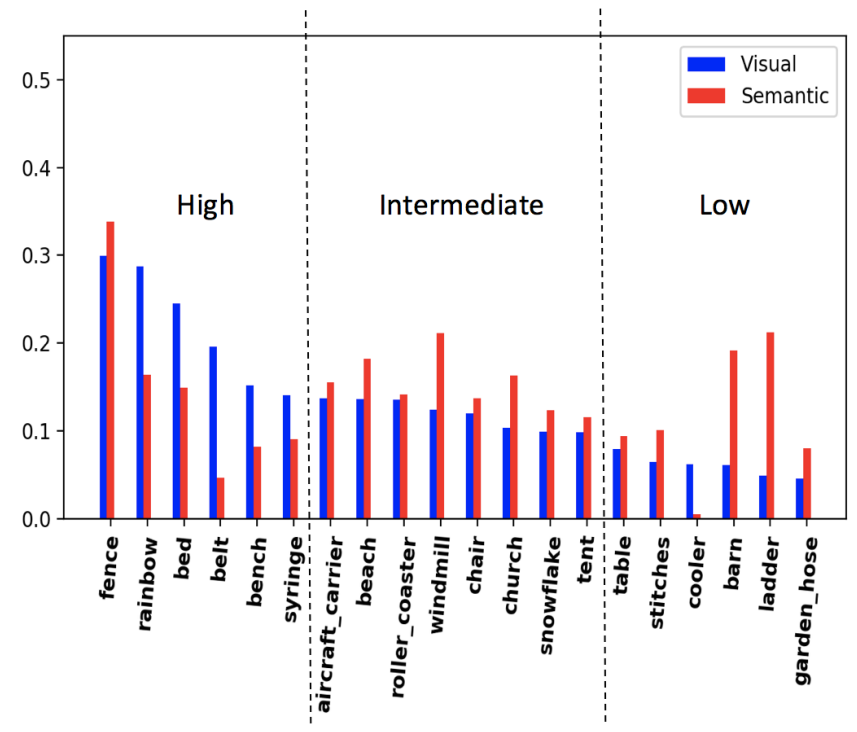

Figure 2. Semantic and visual similarity values between "bridge" category and the top 20 conceptual shift categories. 
1 shows the computational procedure for selecting the target sketch based on the user's input.

\section{EVALUATION STUDY}

We posit that presenting conceptual shifts during the cocreative sketching process supports different types of design creativity. This user study was designed to help understand these variations of design creativity associated with the user's response to conceptual shifts. We investigate how combinatorial, exploratory, and transformational creativity correlate to different degrees of similarity between the user's sketch and the system's response. The degree of similarity is determined in two ways: semantically, by the degree of similarity between words, and visually, by the degree of structural similarity between the two sketches. We hypothesize that high similarity conceptual shift designs are within the expectation of the user, in which the system's response can help the designer to add more details to their initial design. Intermediate similarity conceptual shifts can inspire the designer to explore possible new design ideas associated to their initial design. Lastly, low similarity conceptual shifts have the potential to widen the user's thinking process and help them to add new features from completely a different design space. Hence, we list our hypotheses:

- The user is more likely to produce combinatorial creativity when there is high similarity between the user's sketch and the system's response.

- The user is more likely to produce exploratory creativity when there is intermediate similarity between the user's sketch and the system's response.

- The user is more likely to produce transformational creativity when there is low similarity between the user's sketch and the system's response.

\section{METHOD AND PARTICIPANTS}

The user study included 24 participants recruited from the College of Architecture at a large comprehensive public university in North America, including 7 undergraduate and 17 graduate students. Gender distribution was 15 males and 9 females. The criterion for participating was whether students use sketching often in their design practice. The study utilized a within-subject design where each participant performed a design task in 3 different conditions. We chose three different objects at random from the QD dataset that were appropriate for a design task and randomly assigned them to each condition. The first condition was associated with the design task of sketching a bridge, and the system produced a result that has a high similarity to the participant's sketch. The second condition was associated with the design task of sketching a streetlight, and the system produced a result that had an intermediate similarity to the participant's sketch. The third condition was associated with the design task of sketching a chair, and the system produced a result that has a low similarity to the participant's sketch. Each design task lasted approximately 7 minutes. The order of the three conditions were counterbalanced in order to account for any ordering effects.

We performed a Wizard-of-Oz study to establish a proof of concept before developing a fully integrated system. We used

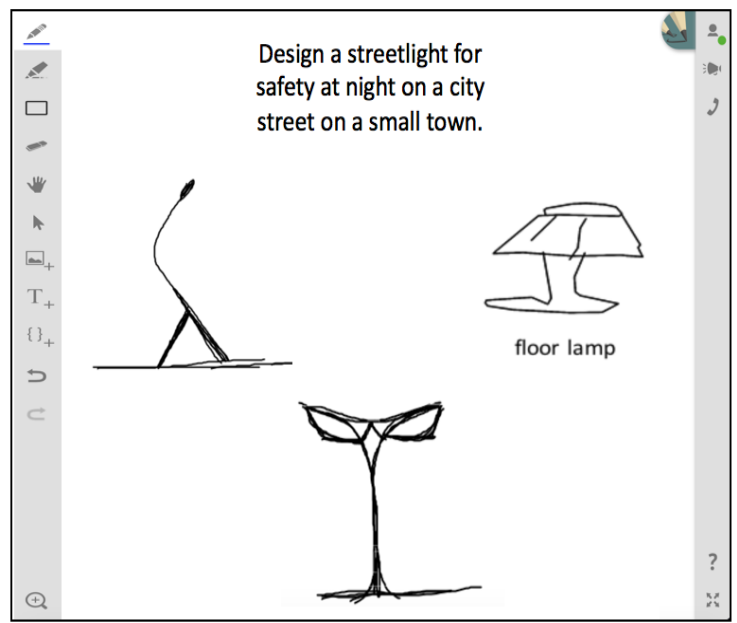

Figure 3. Image of co-creative sketching study interface. Top middle: design task, top left: user's initial design (source sketch), top right: wizard's response (target sketch), and center bottom: user's response (new sketch).

an online sketching tool, called SketchTogether [1], that enables multiple users to contribute to a shared canvas. This platform enabled a human wizard to utilize the results of the conceptual shift algorithm for determining high, intermediate, and low similarity sketches. In this approach, the wizard performed the interaction of placing the selected sketch on the shared canvas. The instructions for the design task for each session are displayed in the middle of the canvas, and the designer is required to respond to the design task by starting to sketch at the top left of the canvas. Then, the system's response (generated by the wizard) is shown at the top right of the canvas. Based on the inspiration from the system's response, the designer is asked to respond by sketching an iteration of their initial sketch at the bottom-center of the canvas. Figure 3 shows the experimental setup of the co-creative sketching tool.

Participants are first introduced to the interface of the sketch tool that they have to interact with during each session. The tasks of the entire session, along with the time frame corresponding to each, are described to the participants. Then, they are asked to perform a training task to ensure their ability to create a sketch and to respond to the wizard's generated sketch with another iteration of their initial design as well as to vocalize the rationale for their design decisions. Moreover, they are told that the goal of the study is to understand how the system's response affects their design process and that they do not need to produce a final design. The entire training session took between 5 to 10 minutes.

After training, participants are required to start the first design task followed by a two-minute break before the second design task begins. The wizard was located in a separate room to respond to the user's sketch in a turn-taking manner. Each design task instruction has two components, including the object label and a context for designing that object, such as "Design a bridge for cars that goes over a large river in the mountains." 


\begin{tabular}{|l|l|l|}
\hline \multicolumn{1}{|c|}{ Combinatorial } & \multicolumn{1}{|c|}{ Exploratory } & \multicolumn{1}{|c|}{ Transformational } \\
\hline $\begin{array}{l}\text { Preserving the original form of the } \\
\text { source sketch while adding elements } \\
\text { from the target sketch }\end{array}$ & $\begin{array}{l}\text { Changing the values for some fea- } \\
\text { tures related to the source sketch } \\
\text { based on values in the target sketch }\end{array}$ & $\begin{array}{l}\text { Changing the overall design of the } \\
\text { source sketch by adding new features } \\
\text { from the target sketch }\end{array}$ \\
\hline $\begin{array}{l}\text { Combine all the features of the source } \\
\text { sketch with all the features of the target } \\
\text { sketch }\end{array}$ & $\begin{array}{l}\text { Explore creating specific patterns in the } \\
\text { source sketch based on the target sketch } \\
\text { (e.g. symmetries, repetition) }\end{array}$ & $\begin{array}{l}\text { Transform the shape contour of the } \\
\text { source sketch based on features from the } \\
\text { target sketch }\end{array}$ \\
\hline $\begin{array}{l}\text { Combine some features of the source } \\
\text { sketch with all the features of the target } \\
\text { sketch }\end{array}$ & $\begin{array}{l}\text { Explore changing the orientation in the } \\
\text { source sketch based on the target sketch } \\
\text { (e.g. angle, direction) }\end{array}$ & $\begin{array}{l}\text { Transform the function of the source } \\
\text { sketch based on features from the target } \\
\text { sketch }\end{array}$ \\
\hline $\begin{array}{l}\text { Combine all features of the source sketch } \\
\text { with some features of the target sketch }\end{array}$ & $\begin{array}{l}\text { Explore changing the size in the source } \\
\text { sketch based on the target sketch (e.g. } \\
\text { distance) }\end{array}$ & \\
\hline $\begin{array}{l}\text { Combine some features of the source } \\
\text { sketch with some features of the target } \\
\text { sketch }\end{array}$ & & \\
\hline
\end{tabular}

Table 1. A framework for three classes of design creativity: Combinatorial, Exploratory, and Transformatinal.

We provided design tasks for three different categories: bridge, chair, and streetlight. After the last session, we performed a retrospective protocol analysis using a screen recording video that was recorded during the session. We showed the video of the entire session to the users and asked them questions related to their response to the system, which allowed participants to explain their thought process throughout the design session. The semi-structured interview questions we asked are:

- What inspired you to draw this sketch?

- Why did you draw in this way?

- Did you see any relationship between your sketch and the system's response?

The answers to retrospective protocol analysis will be used for qualitative analysis. The entire session for each participant takes approximately 30 minutes.

\section{CET ONTOLOGY}

We present the CET ontology as a coding scheme to study the effects of conceptual shifts on cognitive models of creativity. The aim is to code different types of creativity during a cocreative design session. The CET ontology comprises three types of creativity: combinatorial $(\mathrm{C})$, exploratory $(\mathrm{E})$, and transformational $(\mathrm{T})$. We adapted the three types from the Boden creativity taxonomy [3] and the definitions and examples of creative design from Gero [13]. While Boden provides a broad definition of combinatorial, exploratory and transformational creativity, Gero provides more detail in his definitions and examples. We have operationalized the definitions here for the design task in our conceptual shift study. These three types of creativity do not simply map to less or more of some scalar value of creativity, but it has been suggested that transformational creativity is the most original and potentially impactful $[4,13,30]$.
Combinatorial. Combinatorial creativity concerns "the addition of two sets of design concepts or a subset of them" [13]. Boden describes combinatorial creativity as "combining familiar ideas to make unfamiliar combinations, in which the new combinations can be produced either deliberately or unconsciously" [3]. Gero provides two approaches for combination. One approach is based on including ideas from similar designs (e.g. two chairs), whereas the other approach is based on incorporating ideas from dissimilar designs (e.g. chair and cradle) [13]. One key facet of this type of combinatorial creativity is that "the two newly associated ideas share some inherent conceptual structure" [3]. The conceptual structure of one design concept guides the user's design process by being able to add certain features or elements from it.

Based on the definitions described in the literature, we define combinatorial creativity in a co-creative design system as: Preserving the original form of the source sketch while adding elements from the target sketch. We divide combinatorial into four categories. The first category involves combining all the features of two design concepts together. In this scenario, the designer combines all the features of their initial design (source sketch) with all the features of the system's response (target sketch). The second category concerns combining some features of the two design concepts together. This category refers to a scenario in which the designer combines some elements of the source sketch with some elements of the target sketch. The third and fourth category involve combining some features of one design concept with all the features of another concept. This corresponds to two possible scenarios: one is when the designer combines some features of the source sketch with all the features of the target sketch, and the second is when the designer combines all the features of the source sketch with some features of the target sketch (Table 1).

Exploratory. Exploratory creativity involves changing the applicable ranges of values for variables of a design concept [13]. Gero defines exploratory creativity as a design process 


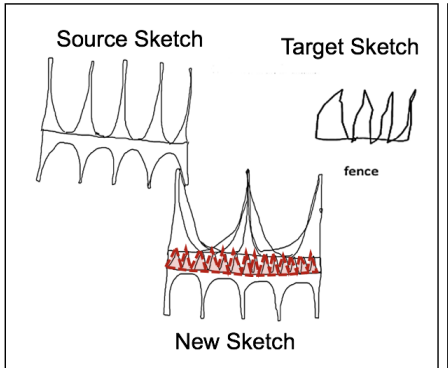

a

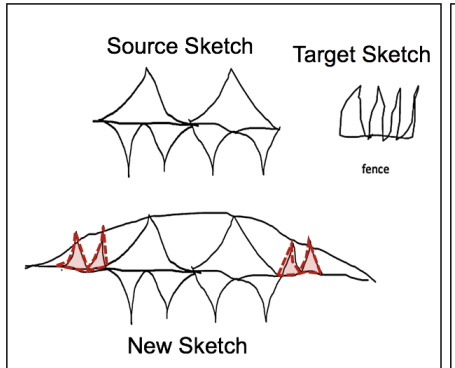

b

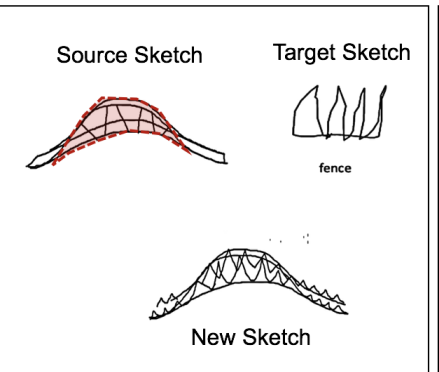

C

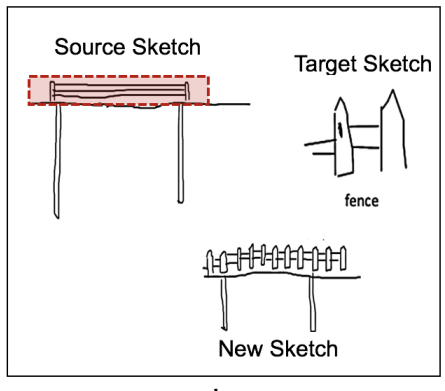

d

Figure 4. The target sketch is applied repeatedly (marked as red) to the source sketch (a). The target sketch is added disjointly (marked as red) to the source sketch (b). Part of the source sketch (marked as red) is replaced with the target sketch (c,d).

in which variable values outside the normal ranges have the potential to introduce unexpected behaviors (e.g. increase the size of wings and the plane becomes too heavy) [13]. Another possible definition of exploratory creativity involves traversing an established conceptual space to create novel and unexpected design ideas [3]. Unlike combinatorial creativity, exploratory corresponds to successive design ideas that are not only novel but also unexpected [3].

Accordingly, we define exploratory creativity in a co-creative design system as: Changing the values of some features related to the source sketch based on values in the target sketch. In this context, features refer to the elements of a design concept (e.g. armrest, backrest, and legs for a chair); whereas values refer to parameters, such as size and angle. We classify exploratory into three categories. The first category explores the creation of specific patterns in the source sketch based on the target sketch. This indicates that specific geometric properties (e.g. symmetrical structures or repetitive patterns) in the target sketch can inspire the designer to create the same structure for the source sketch. The second category explores changing the orientation in the source sketch based on the target sketch. In this scenario, the user changes the angle or the direction of some elements in the source sketch based on inspiration from the target sketch. The last category explores changing the size in the source sketch based on the features in the target sketch. In this case the user changes the size of some elements in the source sketch based on some variable values in the target sketch. Examples are increasing the size of a light post, changing the distance between legs on a chair, and changing the scale of a bridge (Table 1).

Transformational. Transformational creativity involves changing one or more elements of a design concept through the act of developing new ideas [3]. Gero defines transformational creativity as a process in which one or more structure variables of the current design object are altered to produce new variables [13]. Another definition of transformational creativity involves creating new structures through transforming one or more dimension of the current design space [3]. Similar to exploratory creativity, transformational creativity also leads to producing novel and unexpected design ideas [3].

Based on the definitions described above, we define transformational creativity in a co-creative design system as: changing the overall design of the source sketch by adding new features from the target sketch. We consider two possible methods for changing the design of the source sketch. One is when the designer adds new features from another design space. An example of this is when the concept of fluid movement in a speedboat inspires the designer to add wheels to a chair. Another method is when the designer changes the outline shape of a design concept inspired by the structure of another concept, such as changing the shape of a basket from rectangular to circular inspired by the curvy shape of a hat. Accordingly we classify transformational into two different categories. One category involves transforming the shape counter of the source sketch based on the features from the target sketch. The second category concerns transforming the function of the source sketch based on the features from the target sketch (Table 1).

\section{RESULTS}

We employed two independent coders to analyze the retrospective protocol analysis transcripts and the final design sketches for each participant based on the CET framework presented above. The two coders engaged in a blind coding approach where they did not know the condition for each design session. They classified each design session into one of the categories of the CET ontology. This analysis demonstrates the type of design thinking that happened throughout the study.

Combinatorial Creativity. When the concept and the structure of the sketch that is presented to the user (target sketch) is highly similar to the user's initial design (source sketch), the user combines their source sketch with the target sketch in some way (Figures 4 and 5). We assume that this corresponds to combinatorial creativity, as the main features of the source and the target sketch are preserved. There are four possible ways participants could exhibit combinatorial creativity within our study (Table 1). Overall, we observed that all participants engaged in combinatorial creativity while they were in the high similarity condition. We describe those instances below and denote which type of combinatorial creativity they manifested.

Combine all features of the source sketch with all the features of the target sketch

Our data showed that 4/24 participants described their experiences with the system in high similarity mode by combining all features of the source sketch with all the features of the target sketch to create a new design. In the case of P1, P11, 


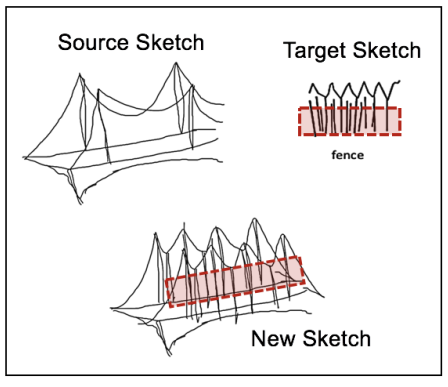

a

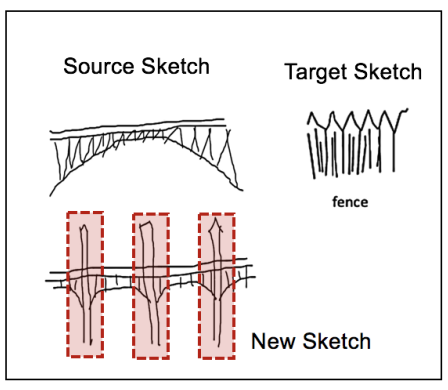

e

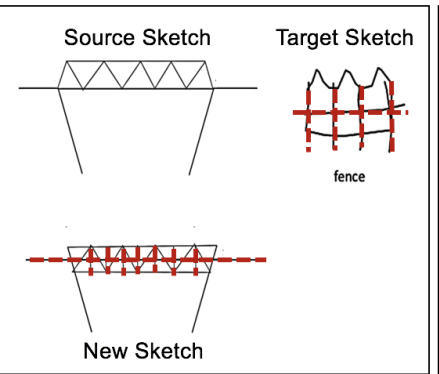

b

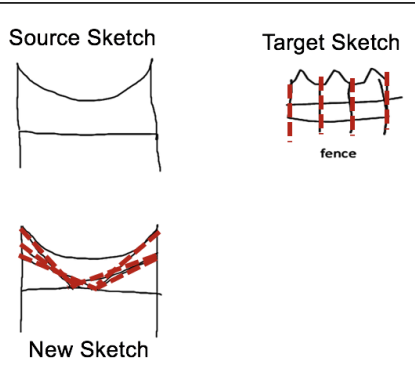

C

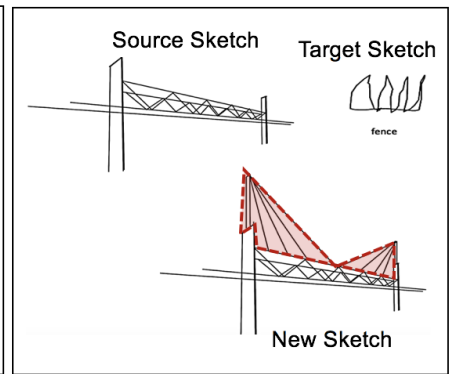

f

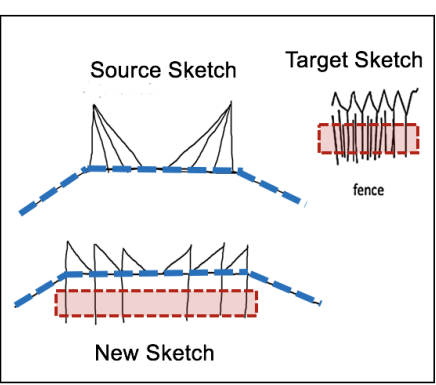

g

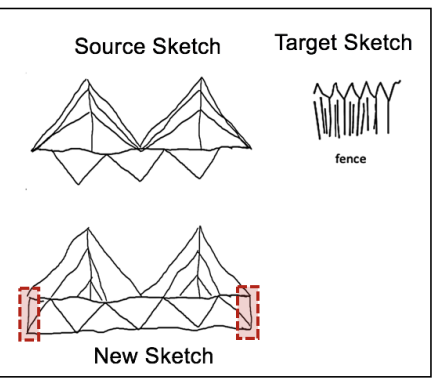

d

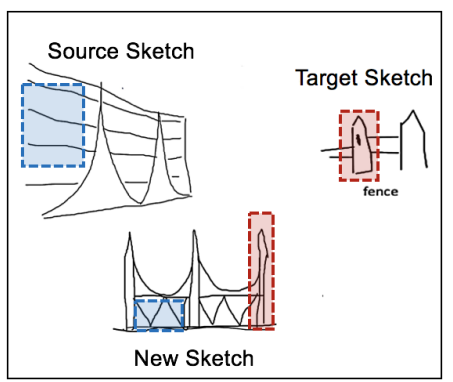

$\mathrm{h}$

Figure 5. Part of the target sketch (marked as red) is applied to the source to add more suspension (a-c). Some elements of the target sketch (marked as red) are applied to the source in order to finalize the structure (d), to uphold the bridge (e), and to add tall posts on top of the bridge (f). Some elements of the source sketch are preserved (marked as blue), while some elements from the target sketch are added to the source (marked as red) (g,h).

P16, the structure of the initial sketch and the target sketch were maintained. In these cases, the target sketch is applied repeatedly to form a part of the source sketch (Figure 4-a). In the case of P13, an extended bridge was designed by dividing the design feature of the target sketch into two equal parts on the same scale and attaching it to both sides of the initial sketch (Figure 4-b). This shows that the way in which the participants applied the target sketch of the fence to their drawings was different. When the design feature of the target sketch was very similar to the initial design (high similarity), the structure of the new design was not significantly different, even though all elements of both sketches were combined in a complex manner.

Combine some features of the source sketch with all the features of the target sketch

This case appeared only twice (2/24) in our dataset. In the case of P10, only the outline of the bridge was maintained, and the red part of Figure 4-c,d was replaced with the target sketch. The structure of the initial design was not changed considerably, although some of the existing design was deleted and replaced with new design elements. Based on the newly combined elements, we can conclude that the new sketch is not a simple morphological transformation, but is instead the emergence of design ideas from the fence, such as protection and safety.

Combine all features of the source sketch with some features of the target sketch

Our data showed that 7/24 participants described their experience with the system in high similarity mode by combining all features of the source sketch with some features of the target sketch to create a new design. This case has two major characteristics. First is to add new design features to the initial sketch as components (4/24). For instance, it is possible to apply a part of the target sketch as it is (Figure 5-a, b) or apply a part of the target sketch after changing the angle or direction (Figure 5-c).

The second major characteristic of this type of combinatorial creativity is to add new structures to existing sketches (3/24). In the case of P17 (Figure 5-d), the initial design shows an unstable bridge. However, a more stable bridge was designed by adding a straight line taken from the target sketch to both ends of the initial sketch. In this case, the structure of the initial design is changed by the inspiration from a part of the target sketch. In the case of P20, pillars were established on the existing design, inspired by the straight line of the fence. Moreover, the large mass was divided into small parts based on the repetitive fence shape (Figure 5-e). In the case of P25, a new shape was also added to the initial design, owing to the inspiration from the sharpness of the fence (Figure 5-f).

Combine some features of the source sketch with some features of the target sketch

Finally, within this category, 11/24 participants described their experience with the system in high similarity mode by combining some features of the source sketch with some features of the target sketch to create a new design. In this case, we found that the structure of the initial design is slightly modified, unlike the previous cases. A suspended bridge shape was designed by moving the baseline upward and arranging triangles, similar to the top part of a fence (Figure 5-g). Alternatively, additional columns were added to the bridge, which mimicked the straight lines of a fence. P4 also actively reflected the design source of the target sketch by abandoning 


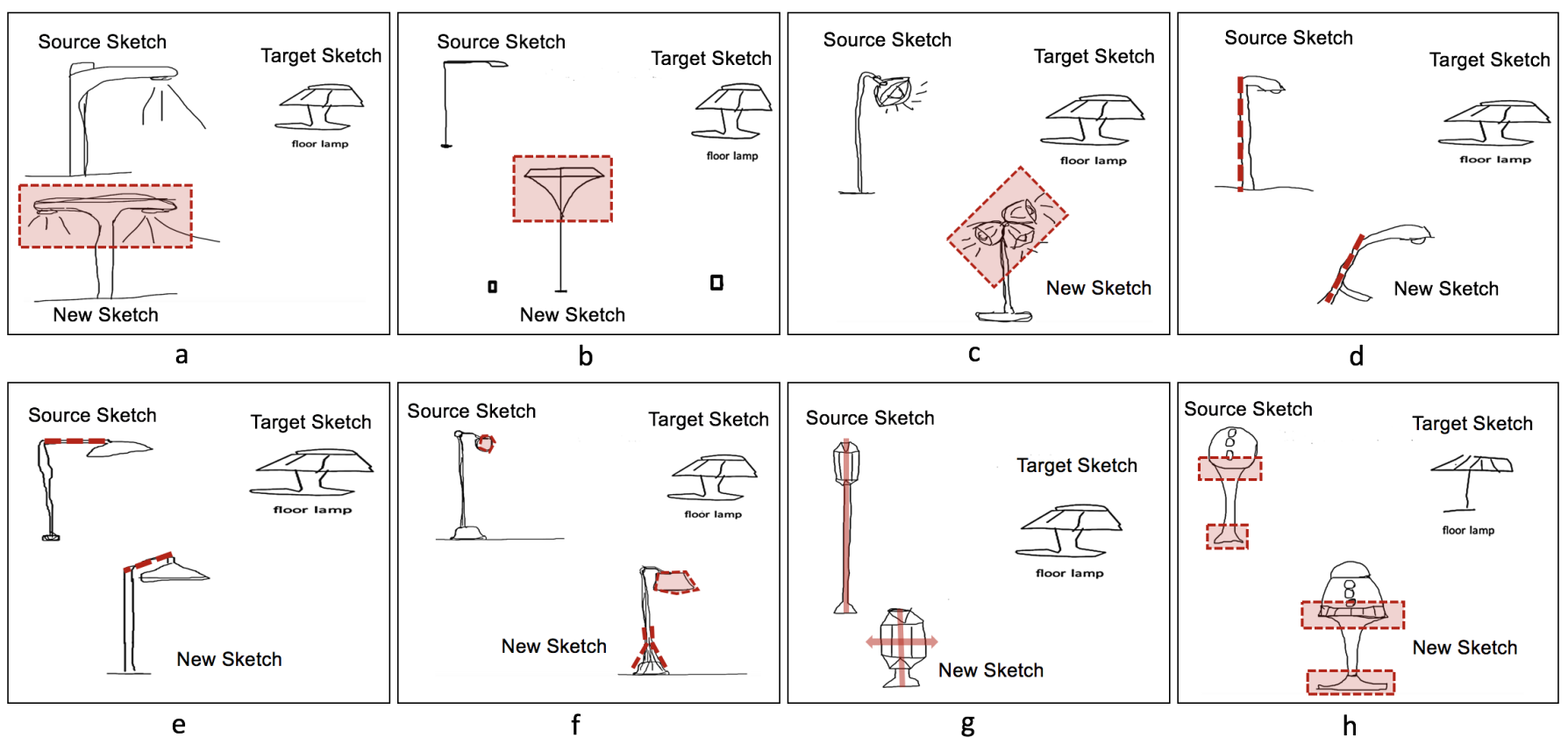

Figure 6. A symmetrical structure (marked as red) is applied to the source sketch (a-c). The orientation of some elements (marked as red) in the source sketch is changed (d,e). The size of some elements (marked as red) in the source sketch is altered (f-h).

the existing style (Figure 5-h). It parallels the structure of the target sketch instead of the initial design.

Exploratory Creativity. When the sketch that is presented to the user (target sketch) shares some structural characteristics with the user's initial design (source sketch) and the concept is of intermediate similarity, the user modifies the source sketch by giving new values to some features of their original design (Figure 6). We assume this corresponds to exploratory creativity because the user is making some changes to the initial design in the same design space. Based on the CET framework, there are three ways participants could demonstrate exploratory creativity within our study (Table 1 ). Our observation shows that $18 / 24$ participants engaged in exploratory creativity while they were in the intermediate similarity condition.

Explore creating specific patterns in the source sketch based on the target sketch

Overall, 8/24 participants described their experience with the system in intermediate similarity mode through creating specific patterns in the source sketch based on the target sketch to create a new design. 7/8 participants who belong to this case were inspired from the symmetric structure of the floor lamp. In these instances, the source sketch was made to be more symmetric by adding another light source to the other side of the light post. Among them, 3/7 participants symmetrically copied the lamp part of the initial sketch as it is without modification (Figure 6-a), whereas 4/7 participants explored new design ideas by changing some design elements that were present in their source sketch. For instance, the size of the lamp part increases, and the top and bottom are reversed (Figure 6-b), or a new type of lamp is designed, as exemplified by
P3's design. In the case of P8 (1/8), the participant does not simply arrange the new lamp symmetrically but develops a new design type by enumerating the design object repetitively (Figure 6-c). In this case, the lamp part of the street light acts as a major element that guides the design, and the pole component is a subsidiary element that informs the length, the angle, and the slope of the light to create balance in the design. It indicates a scenario that explores other design elements to express a major design concept, which is symmetry.

Explore changing the orientation in the source sketch based on the target sketch

This case appeared only twice (2/24) in our dataset, in which new design ideas are explored by adjusting the orientation of some features in the source sketch. The two participants changed the orientation of these altered design elements in the source sketch without significantly changing the type or the shape of the initial sketch (Figure 6-d,e). Both participants responded that they changed the angle of a pole to adjust the angle of the light from the street light, in response to the floor lamp that has a downward focused light. These two cases have the least number of changes in terms of the shape in exploratory creativity.

Explore changing the size in the source sketch based on the target sketch

Lastly, 8/24 participants described their experience with the system in intermediate similarity mode by changing the size of some elements in the source sketch based on the target sketch to create a new design. 3/8 participants enlarged the size of a lamp as the simplest variation. In case of $\mathrm{p} 9$, in addition to increasing the size of the lamp, a gradual size increase was applied to the base of the source sketch inspired 

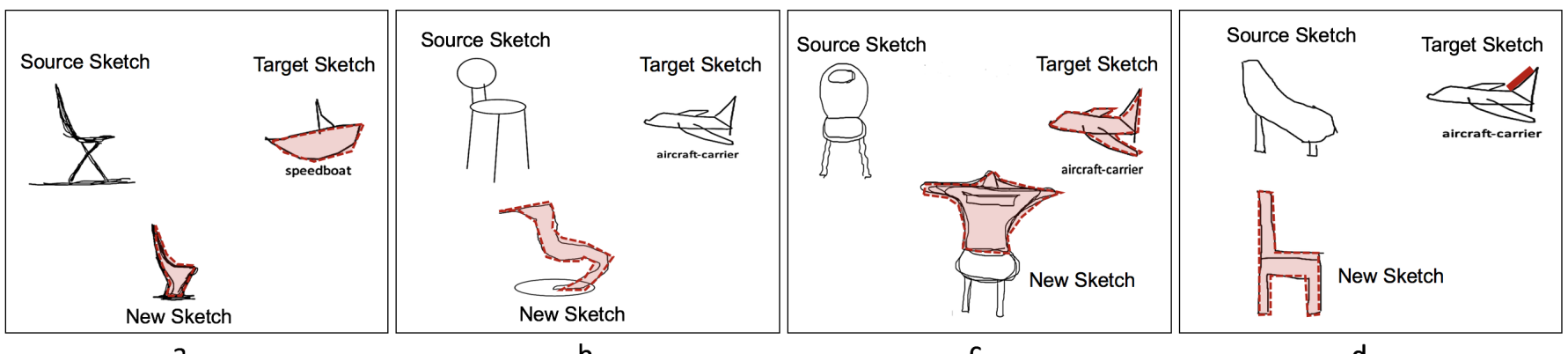

a

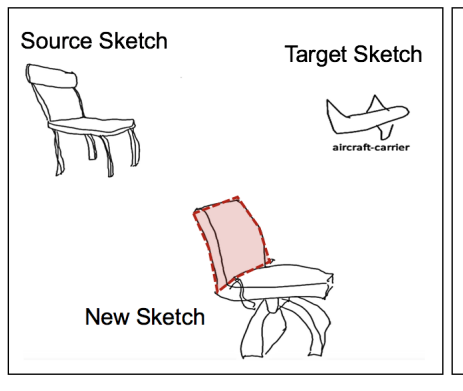

b
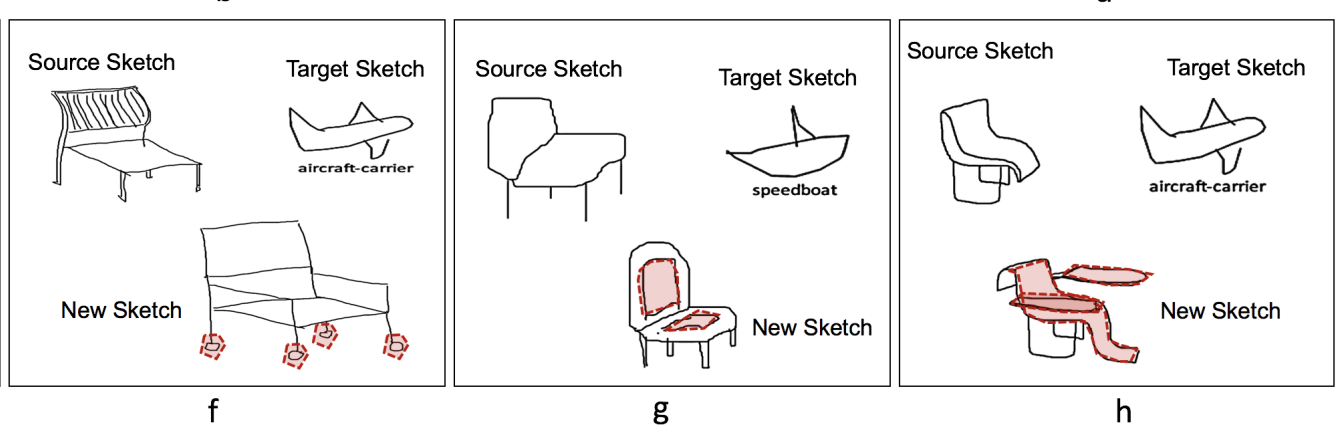

Figure 7. Some or all features of the source sketch structure is transformed (marked as red) to resemble the outline shape of some or all elements in the target sketch (a-d). The function of some elements in the source sketch is transformed (marked as red) or new functions (marked as red) are added (e-h).

by the gradual change in the base of the floor lamp (Figure 6-f). 2/8 participants changed their initial sketch by reducing the total height of the street light. In the case of P12, the initial sketch was changed to reflect the scale of the target sketch by reducing total height and increasing the size of the lamp component (Figure 6-g). In the cases of P1 and P16, they showed a gradual change while several design elements act in combination. In the case of $\mathrm{P} 1$, the round shape of the source sketch is made more angular by including elements from the target sketch (Figure 6-h). The support of the lamp shows a shape that mixes the source sketch and the target sketch to extend both ends of the base.

Exploratory and Combinatorial Creativity. In some instances, when the system is in intermediate similarity mode, the user adds some of the elements from the target sketch, while at the same time changes some features of their initial design (Figure 8-a,b). We assume this corresponds to both exploratory and combinatorial creativity, as the user is utilizing both types of design creativity. Overall, we found that $4 / 24$ participants described their experience with the system in intermediate similarity mode through changing some features of their initial design and combining them with the system's response. Three of the participants created a symmetric light shade design that took inspiration from the symmetry present in the target sketch (Figure 8-a). Another participant increased and expanded the lamp size to resemble an LED light, while at the same time incorporated the lamp shade in the new design (Figure 8-b).

Transformational Creativity. When the concept and the structure of the sketch that is presented to the user (target sketch) is less similar to the user's initial design (source sketch), we observe that the user modifies the source sketch based on features and elements from the target sketch (Figure 7). In other words, the user moves from the current design space into another design space by searching alternative structures of the source sketch in the new design space. We assume this corresponds to transformational creativity, as the user is changing the design space completely. Based on the CET framework, there are two ways participants could exhibit transformational creativity within our study (Table 1). Our dataset showed that all participants engaged in transformational creativity when they were in the low similarity condition.

Transform the shape contour of the source sketch based on features from the target sketch

Our results showed that $13 / 24$ participants described their experience with the system in low similarity mode by transforming the shape contour of the source sketch based on features from the target sketch to create a new design. We found 12/13 participants were inspired by the entire shape rather than a part of the target sketch, and they applied it to the source sketch. All participants applied the curvy shape of the speedboat and aircraft-carrier sketches provided by the system to their designs. Among the participants, 6/12 demonstrated that the curvy shape of the target sketch was used to transform the entire outline of the source sketch into a streamlined shape. Additionally, there was a participant that maintained the overall shape of their initial drawing while changing some contours to be curvy like the target sketch (Figure 7-a), and creating a totally new shape significantly departing from the existing shape (Figure 7-b). On the other hand, 6/12 participants showed the case of applying a streamlined element of the target sketch to only a part of the source sketch (Figure 7-c). In the case 


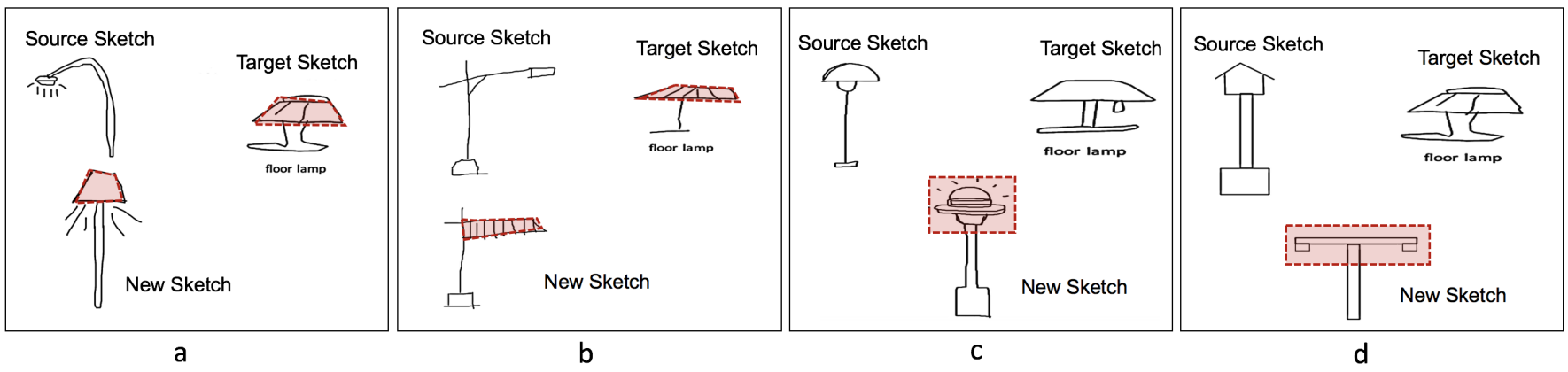

Figure 8. A symmetric position in the center is applied while adding the lamp shade (marked as red) to the source sketch (a). The size of the lamp is increased and concurrently the lamp shade is added (marked as red) (b). A part of the source sketch is transformed (marked as red) (c,d).

of p19, the straight-lined elements of the aircraft inspired and transformed the user's design. There was initially a curvy chair that was transformed into a rectangular form by adding a straight-lined element (Figure 7-d).

Transform the function of the source sketch based on features from the target sketch

Finally, 11/24 participants described their experience with the system in low similarity mode through transforming the function of the source sketch based on features from the target sketch to create a new design. In this case, the design idea is obtained from the function and/or the structure of the target sketch. For example, one participant utilized the form and function of an aircraft seat to transform their chair design to a reclining chair that is more comfortable (Figure 7-e). In another instance, the participant was inspired by the movement of the aircraft in the air, and added wheels to the chair to make it movable (Figure 7-f). By recalling the cushion feature of a chair installed inside the speedboat (target sketch), a participant transformed their design to include this function (Figure 7-g). In 7 instances, the entire outline of the initial sketch is transformed to add armrests inspired by the wings of the aircraft (target sketch) (Figure 7-h).

Transformational creativity also emerged twice (2/24) when participants were experiencing the intermediate similarity condition. When the system is in intermediate similarity mode, the user typically modifies the source sketch by giving new values to some features of their original design. However, in this case, the participants added a new design element to the source sketch by getting a new idea from the functions of the target sketch. These instances are not combinatorial nor exploratory creativity because they did not utilize elements of the target sketch or modify the parameters of existing values from the target sketch, instead the participants developed a new design with a transformed shape (Figure 8-c,d).

\section{LIMITATION AND FUTURE WORK}

One limitation of our study design is associating each object to a single experimental condition. While the results show that sketches of an object can have different structures and therefore belong to different clusters in our AI model, having the same semantic component in each condition could still create a bias among participants. Another limitation of the current study was qualitative nature of the analysis. Future studies will randomly vary the objects presented in each condition and use statistical measures such as chi-squared with more participants to establish the statistical significance of the results.

Our next step is to develop an integrated sketching interface and AI system. The interface will have a sketching pane for both the user and system and two sliders for changing the parameters of the system: one for adjusting visual similarity and the second for changing the semantic similarity. Such a system will enable us to study the correlation between the degree of similarity and the three types of creativity more quantitatively. Moreover, the system will allow the study of the user behaviour during co-creation, such as changing sliders, erasures, amount of ink used, and the number of sketches created.

\section{CONCLUSION}

This paper describes a computational model of conceptual shift for a co-creative sketching tool to encourage design creativity. The algorithm selects concepts that have high, intermediate, and low visual and semantic similarity to the designer's sketch and presents these objects to the user on the shared canvas. Visual similarity entails identifying clusters of sketch categories that share structural characteristics to the user's input. Semantic similarity determines the degree of similarity between the user's sketch and the selected category names. We have presented initial evidence to support our hypothesis that high, intermediate, and low similarity are associated with combinatorial, exploratory, and transformational creativity, respectively. We introduce a framework for these types of creativity as a general coding scheme to analyze the user's design thinking process. The findings suggest that the high similarity condition may help designers combine two design concepts, the intermediate similarity condition helps designers change the range of values associated with some variables of their initial design, and the low similarity condition may inspire designers to add new features from another design space to their initial design.

\section{REFERENCES}

[1] 2019. SketchTogether. (2019). https://sketchtogether.com 
[2] John A Biles and others. 1994. GenJam: A genetic algorithm for generating jazz solos. In ICMC, Vol. 94. 131-137.

[3] Margaret A Boden. 1996. Creativity. In Artificial Intelligence. Elsevier, 267-291.

[4] Margaret A Boden. 2004. The creative mind: Myths and mechanisms. Routledge.

[5] V. Carbune. 2017. Recurrent Neural Networks for Drawing Classification. (2017). https://www. tensorflow. org/tutorials/sequences/recurrent_quickdraw

[6] Simon Colton, Geraint A Wiggins, and others. 2012. Computational creativity: The final frontier?. In Ecai, Vol. 2012. Montpelier, 21-16.

[7] Jim Davies, Ashok K Goel, and Nancy J Nersessian. 2009. A computational model of visual analogies in design. Cognitive Systems Research 10, 3 (2009), 204-215.

[8] Nicholas Davis. 2013. Human-computer co-creativity: Blending human and computational creativity. In Ninth Artificial Intelligence and Interactive Digital Entertainment Conference.

[9] Nicholas Davis, Chih-PIn Hsiao, Kunwar Yashraj Singh, Lisa Li, Sanat Moningi, and Brian Magerko. 2015. Drawing apprentice: An enactive co-creative agent for artistic collaboration. In Proceedings of the 2015 ACM SIGCHI Conference on Creativity and Cognition. ACM, 185-186.

[10] Nicholas Davis, Chih-PIn Hsiao, Kunwar Yashraj Singh, Lisa Li, and Brian Magerko. 2016. Empirically studying participatory sense-making in abstract drawing with a co-creative cognitive agent. In Proceedings of the 21 st International Conference on Intelligent User Interfaces. ACM, 196-207.

[11] Ernest A Edmonds, Alastair Weakley, Linda Candy, Mark Fell, Roger Knott, and Sandra Pauletto. 2005. The studio as laboratory: combining creative practice and digital technology research. International Journal of Human-Computer Studies 63, 4-5 (2005), 452-481.

[12] Dedre Gentner. 1983. Structure-mapping: A theoretical framework for analogy. Cognitive science 7, 2 (1983), $155-170$.

[13] John S Gero. 2000. Computational models of innovative and creative design processes. Technological forecasting and social change 64, 2-3 (2000), 183-196.

[14] Guy Hoffman and Gil Weinberg. 2010. Gesture-based human-robot jazz improvisation. In Robotics and Automation (ICRA), 2010 IEEE International Conference on. IEEE, 582-587.

[15] Mikhail Jacob, Gaëtan Coisne, Akshay Gupta, Ivan Sysoev, Gaurav Gav Verma, and Brian Magerko. 2013. Viewpoints ai. In Ninth Artificial Intelligence and Interactive Digital Entertainment Conference.
[16] Jonas Jongejan, Henry Rowley, Takashi Kawashima, Jongmin Kim, and Nick Fox-Gieg. 2016. The quick, draw!-ai experiment. (2016).

[17] Pegah Karimi, Nicholas Davis, Kazjon Grace, and Mary Lou Maher. 2018a. Deep Learning for Identifying Potential Conceptual Shifts for Co-creative Drawing. arXiv preprint arXiv:1801.00723 (2018).

[18] Pegah Karimi, Kazjon Grace, Nicholas Davis, and Mary Lou Maher. 2018b. Creative Sketching Apprentice: Supporting Conceptual Shifts in Sketch Ideation. In International Conference on-Design Computing and Cognition. Springer, 721-738.

[19] Pegah Karimi, Mary Lou Maher, Kazjon Grace, and Nicholas Davis. 2018c. A computational model for visual conceptual blends. IBM Journal of Research and Development (2018).

[20] Antonios Liapis, Georgios N Yannakakis, and Julian Togelius. 2013. Sentient Sketchbook: Computer-aided game level authoring.. In FDG. 213-220.

[21] Todd Lubart. 2005. How can computers be partners in the creative process: classification and commentary on the special issue. International Journal of Human-Computer Studies 63, 4-5 (2005), 365-369.

[22] Pedro Lucas and Carlos Martinho. 2017. Stay Awhile and Listen to 3Buddy, a Co-creative Level Design Support Tool. In ighth International Conference on Computational Creativity, ICCC, Atlanta.

[23] Tomas Mikolov. 2016. word2vec: Tool for computing continuous distributed representations of words. https://code.google. com/p/word2vec. (2016).

[24] David Norton, Derrall Heath, and Dan Ventura. 2014. Autonomously Managing Competing Objectives to Improve the Creation and Curation of Artifacts.. In ICCC. 23-32.

[25] R Keith Sawyer and Stacy DeZutter. 2009. Distributed creativity: How collective creations emerge from collaboration. Psychology of aesthetics, creativity, and the arts 3, 2 (2009), 81.

[26] Ben Shneiderman. 2007. Creativity support tools: Accelerating discovery and innovation. Commun. ACM 50, 12 (2007), 20-32.

[27] Ben Shneiderman, Gerhard Fischer, Mary Czerwinski, Mitch Resnick, Brad Myers, Linda Candy, Ernest Edmonds, Mike Eisenberg, Elisa Giaccardi, Tom Hewett, and others. 2006. Creativity support tools: Report from a US National Science Foundation sponsored workshop. International Journal of Human-Computer Interaction 20, 2 (2006), 61-77.

[28] Masaki Suwa and Barbara Tversky. 1997. What do architects and students perceive in their design sketches? A protocol analysis. Design studies 18, 4 (1997), 385-403. 
[29] Matthias Voigt, Björn Niehaves, and Jörg Becker. 2012. Towards a unified design theory for creativity support systems. In International Conference on Design Science Research in Information Systems. Springer, 152-173.
[30] Geraint A Wiggins. 2006a. A preliminary framework for description, analysis and comparison of creative systems. Knowledge-Based Systems 19, 7 (2006), 449-458.

[31] Geraint A Wiggins. 2006b. Searching for computational creativity. New Generation Computing 24, 3 (2006), 209-222. 